\title{
Hyperurikämie und Gicht
}

\author{
Wolfgang Gröbner
}

Die Gicht gehört zu den häufigsten Gelenkerkrankungen - etwa 4-5\% aller Männer, die das 65. Lebensjahr erreichen, erleiden einen Gichtanfall. Dieser ist äußerst schmerzhaft: Jede Bewegung oder Erschütterung kann unerträglich werden. Nicht nur wegen der Schmerzen ist bei einem Anfall schnelles Handeln gefragt - ein Therapiebeginn später als 24 Stunden nach Symptombeginn hat geringere Erfolgsaussichten.

\section{Der konkrete Fall}

Dekompensierte Herzinsuffizienz | Ein 58-jähriger übergewichtiger Patient $(170 \mathrm{~cm}, 82 \mathrm{~kg})$ wird gegen Abend mit Atemnot und Beinödemen in die Klinik eingeliefert. Bei bereits bekannter koronarer 3-Gefäß-Erkrankung diagnostiziert man eine dekompensierte Herzinsuffizienz. Auskultatorisch finden sich über der Lunge beidseits basal nicht klingende Rasselgeräusche. Der Blutdruck beträgt 180/100 mmHg.

Laborbefunde bei Aufnahme | Leukozyten 9000/ $\mu \mathrm{l}, \mathrm{Hb} 12,5 \mathrm{~g} / \mathrm{dl}$, Thrombozyten $250000 / \mu \mathrm{l}$, Serum-Harnsäure 7,5 mg/ dl (450 $\mu \mathrm{mol} / \mathrm{l})$, SGOT 40 U/1, SGPT 60 U/1, Gamma-GT 70 U/1. Die Nierenretentionswerte und Serum-Elektrolyte liegen im Normbereich, es findet sich eine mäßige Hypercholesterinämie und Hypertriglyzeridämie.

Therapie mit Furosemid | Eine Therapie mit einem ACE-Hemmer und einem Schleifendiuretikum wird eingeleitet - initial wird 20 mg Furosemid i.v. verabreicht. In der Nacht wacht der Patient mit heftigen Schmerzen im Bereich des linken Großzehengrundgelenks auf. Die Schmerzen sind so stark, dass er den Druck der Bettdecke oder Erschütterungen im Raum kaum ertragen kann. Die symptombezogene klinische Untersuchung ergibt eine ausgeprägte Schwellung und Rötung des linken Großzehengrundgelenks, die die Gelenkgrenzen überschreitet. Anamnestisch gibt der Patient an, dass eine Monarthritis des rechten Sprunggelenks vor Jahren ohne medikamentöse Therapie abgeklungen sei.

Colchicin | Unter dem Verdacht auf einen Gichtanfall leitet man umgehend eine Therapie mit Colchicin ein. Die am nächsten Morgen bestimmte Serum-Harnsäure beträgt $8,6 \mathrm{mg} / \mathrm{dl} \quad(516$ $\mu \mathrm{mol} / \mathrm{l}$ ); die Leukozyten liegen bei $15300 / \mu l$. Die Röntgenaufnahme der Vorfüße ergibt keinen pathologischen Befund.

Saluretika-induzierter Gichtanfall | Aufgrund des typischen lokalen Befunds, des Beschwerdebilds und der erhöhten Serum-Harnsäurekonzentration diagnostizierte man einen Saluretika-induzierten Gichtanfall. Der Patient erhielt unmittelbar nach Beginn der Symptomatik 1,0 mg Colchicin per os, $1 \mathrm{~h}$ später nochmals $0,5 \mathrm{mg}$. Daraufhin ließen die Schmerzen deutlich nach. Ab dem Folgetag wurde Colchicin in einer Dosis von $2 \times 0,5 \mathrm{mg}$ täglich per os verabreicht. Die nochmals bestimmte Serum-Harnsäure ergab 3 Tage später einen Wert von $8,5 \mathrm{mg} / \mathrm{dl}(510 \mu \mathrm{mol} / \mathrm{L})$.

Therapie mit Allopurinol | Nach Abklingen des akuten Gichtanfalls setzte man zur dauerhaften Senkung der Serum-Harnsäure neben diätetischen Maßnahmen Allopurinol ein, beginnend mit $100 \mathrm{mg}$ täglich. Angestrebt wurde eine Serum-Harnsäurekonzentration $<6,0 \mathrm{mg} / \mathrm{dl} \quad(360$ $\mu \mathrm{mol} / \mathrm{l})$. Unter einer Dosis von $300 \mathrm{mg}$ Allopurinol täglich lag die Serum-Harnsäurekonzentration schließlich zwischen 5,5 (330 $\mu \mathrm{mol} / \mathrm{l})$ und $6,0 \mathrm{mg} / \mathrm{dl}(360 \mu \mathrm{mol} / \mathrm{l})$. Als Anfallsprophylaxe erhielt der Patient die bereits angesetzte ColchicinTherapie mit $2 \times 0,5 \mathrm{mg}$ täglich über insgesamt 3 Monate. Weitere Gichtanfälle traten unter konsequenter harnsäuresenkender Therapie nicht mehr auf.

\section{Gicht}

Definition I Die Gicht ist eine Erkrankung, die meist als akute Monarthritis beginnt und nach symptomfreiem Intervall rezidiviert. Allmählich geht die Erkrankung dann in eine chronische destruierende Gelenkerkrankung über.

Ursache der Gicht ist in den meisten Fällen eine angeborene Stoffwechselstörung, die familiäre Hyperurikämie.

Neben der akuten und chronischen Arthritis sind weitere klinische Manifestationen einer Hyperurikämie

- Tophi,

- Gichtgeschwüre, 
- Uratnephropathie mit Niereninsuffizienz und Hypertonie sowie

- Harnsäurenephrolithiasis.

Zunehmende Häufigkeit | Die Häufigkeit der Hyperurikämie und Gicht hat in den letzten zwei Jahrzehnten deutlich zugenommen. Vermutete Ursachen sind

- die längere Lebenserwartung,

- die Zunahme von Adipositas und metabolischem Syndrom,

- eine erhöhte Prävalenz von Patienten mit fortgeschrittener Niereninsuffizienz,

- der vermehrte Einsatz von Saluretika und niedrig dosierter Acetylsalicylsäure sowie

- die Zunahme von Organtransplantationen und der damit verbundene Einsatz von Cyclosporin A.

Prävalenz | Die Gicht zählt mit einer Prävalenz von $1,4 \%$ zu den häufigsten entzündlichen Gelenkerkrankungen [1]. Männer sind häufiger betroffen als Frauen, da Frauen vor der Menopause durch die urikosurische Wirkung von Östrogenen weitgehend geschützt sind. Man kann davon ausgehen, dass etwa 4-5\% aller Männer, die das 65. Lebensjahr erreichen, einen Gichtanfall erleiden. Bei den stationären Patienten eines Kreiskrankenhauses beobachteten wir eine Hyperurikämie bei 36\% der Männer und bei 23 \% der Frauen - ein großer Prozentsatz der Bevölkerung ist dem Risiko von klinischen Komplikationen einer Hyperurikämie ausgesetzt [2].

\section{Normaler Serumharnsäure-Spiegel und Hyperurikämie}

Einflussfaktoren auf die Harnsäure | Alter, Geschlecht und Ernährung sind die wesentlichen Faktoren, die die Serumharnsäure-Konzentration des Gesunden beeinflussen. Überprüft man die Verteilungskurven der Harnsäurewerte im Serum aus großen epidemiologischen Untersuchungsreihen, so erlauben sie mit statistischen Methoden keine eindeutige Abgrenzung von normalen und erhöhten Werten. Dies beruht u.a. darauf, dass die Werte der Harnsäure ernährungsabängig sind.
Unter Berücksichtigung der Löslichkeitsgrenze von Natriumurat im Plasma, kann man bei Männern und Frauen die Hyperurikämie als eine Serumharnsäure-Konzentration oberhalb $6,4 \mathrm{mg} / \mathrm{dl}(384 \mu \mathrm{mol} / \mathrm{l})$ definieren.

Das Überschreiten des Löslichkeitsprodukts der Harnsäure geschieht z. B. durch

- vermehrte Zufuhr von Nahrungspurinen,

- Alkoholexzess,

- niedrige Körpertemperatur oder

- Verschlechterung der Nierenfunktion.

Dies kann zur Ausfällung von Uratkristallen und damit zu klinischen Manifestationen führen.

\section{Pathogenese der Hyperurikämie und ihrer klinischen Manifestationen}

Eine Hyperurikämie entsteht, wenn Harnsäure vermehrt gebildet oder vermindert ausgeschieden wird. In seltenen Fällen kombinieren sich beide Mechanismen.

Primäre Hyperurikämie I Die primäre (familiäre) Hyperurikämie beruht bei der Mehrzahl aller Patienten auf einer Störung der renalen Harnsäureausscheidung (ca. 98-99\%). Die genetische Grundlage konnte teilweise aufgeklärt werden [3]. Eine vermehrte endogene Harnsäuresynthese infolge von Enzymdefekten des Purinstoffwechsels wird lediglich bei $1-2 \%$ aller Patienten beobachtet (verminderte Aktivität der HypoxanthinGuanin-Phosphoribosyltransferase = KelleySeegmiller-Syndrom und Überaktivität der Phosphoribosylpyrophosphat-Synthetase) [4]. Von diesen primären Hyperurikämien sind sekundäre Formen abzugrenzen ( $\bullet$ Tab. 1 )

Entstehung des akuten Gichtanfalls | Bei physiologischem pH liegt Harnsäure praktisch vollständig als Natriumurat vor. Überschreitet die Natriumurat-Konzentration die physiko-chemische Löslichkeitsgrenze von $6,4 \mathrm{mg} / \mathrm{dl}(384 \mu \mathrm{mol} / 1)$, erhöht sich das Risiko für das Ausfallen der Substanz. Werden dabei phagozytierbare Mikrokristalle gebildet, kommt es zum akuten Gichtanfall.
Tab. 1 Wichtige Ursachen (und Beispiele) sekundärer Hyperurikämien. Bei den eingeklammerten Angaben spielen für das Zustandekommen einer Gicht wahrscheinlich hereditäre Faktoren ebenfalls eine Rolle. vermehrte Harnsäurebildung

- chronische myeloische Leukämie

- Polyzythämia vera

- Osteomyelofibrose

- (sekundäre Polyglobulie bei Herz- und Lungenkrankheiten)

- (hämolytische Anämie)

- Glukose-6-Phosphatase-Mangel

- zytostatische Therapie

- Bestrahlung verminderte renale Harnsäureausscheidung

- Nierenkrankheiten

- Ketoazidose: Fasten, entgleister Diabetes mellitus

- Hyperlaktazidämien: hoher Alkoholspiegel, Glukose-6-Phosphatase-Mangel

- Arzneimittel, z. B. Saluretika, Cyclosporin A, Pyrazinamid

- Vergiftung (Blei) 
Die pathogenetischen Vorstellungen beim akuten Gichtanfall sind in $>$ Abb. 1 dargestellt [5]. Uratkristalle werden von Makrophagen phagozytiert und aktivieren dann den NALP3-InflammasomProteinkomplex. Es kommt zur Freisetzung von Interleukin-1 Beta (IL-1 Beta) aus einem Vorläuferpeptid. Interleukin-1 Beta bindet an den Interleukin-1-Rezeptor auf Endothel- und Synovialzellen. Diese Zellen werden zur Produktion proinflammatorischer Zytokine und Chemokine stimuliert. Dadurch werden neutrophile Granulozyten rekrutiert, die verschiedene Entzündungsmediatoren freisetzen. So läuft ein Circulus vitiosus immer schneller ab, bis der Höhepunkt der Entzündung erreicht ist. Bei chronischer Ablagerung von Urat entstehen Tophi.

Typische Manifestationsorte für Gichtanfälle sind Gewebe mit geringer Durchblutung und / oder niedriger Körpertemperatur. Häufig sind Gichtanfälle auch in vorgeschädigten arthrotischen Gelenken zu beobachten.

Harnsäurenephrolithiasis | Nicht selten tritt bei Gicht eine Harnsäurenephrolithiasis auf. Risikofaktoren sind

- eine erhöhte renale Harnsäureausscheidung,

- ein niedriges Harnvolumen und

- ein erniedrigter Urin-pH-Wert.

Niereninsuffizienz | Auch eine Niereninsuffizienz ist bei Gichtkranken nicht selten. Sie reflektiert meist die Koexistenz anderer Erkrankungen wie Hypertonie, Diabetes mellitus und Arteriosklerose. Wird die renale Harnsäureausscheidung akut gesteigert, z.B. im Rahmen der zytostatischen Therapie eines hochmalignen Lymphoms, kann es zum akuten Nierenversagen kommen.

\section{Klinik der Gicht}

Verlauf I Klinisch manifestiert sich eine Hyperurikämie in:

- akuter Arthritis, Sehnenscheidenentzündung, Bursitis

- chronisch deformierender Arthritis mit periartikulären und subkutanen Harnsäureablagerungen (Tophi)

- renalen Komplikationen, z. B. Harnsäurenephrolithiasis

Unbehandelt nimmt die Gicht einen Verlauf, den man in vier Stadien einteilen kann:

1. die asymptomatische Hyperurikämie

2. den akuten Gichtanfall

3. die interkritische Gicht (symptomfreies Intervall zwischen den Anfällen)

4. die chronisch, tophöse Gicht

Akuter Anfall | Das erste klinische Symptom einer Hyperurikämie ist meist der akute Gichtanfall. Charakteristisch ist

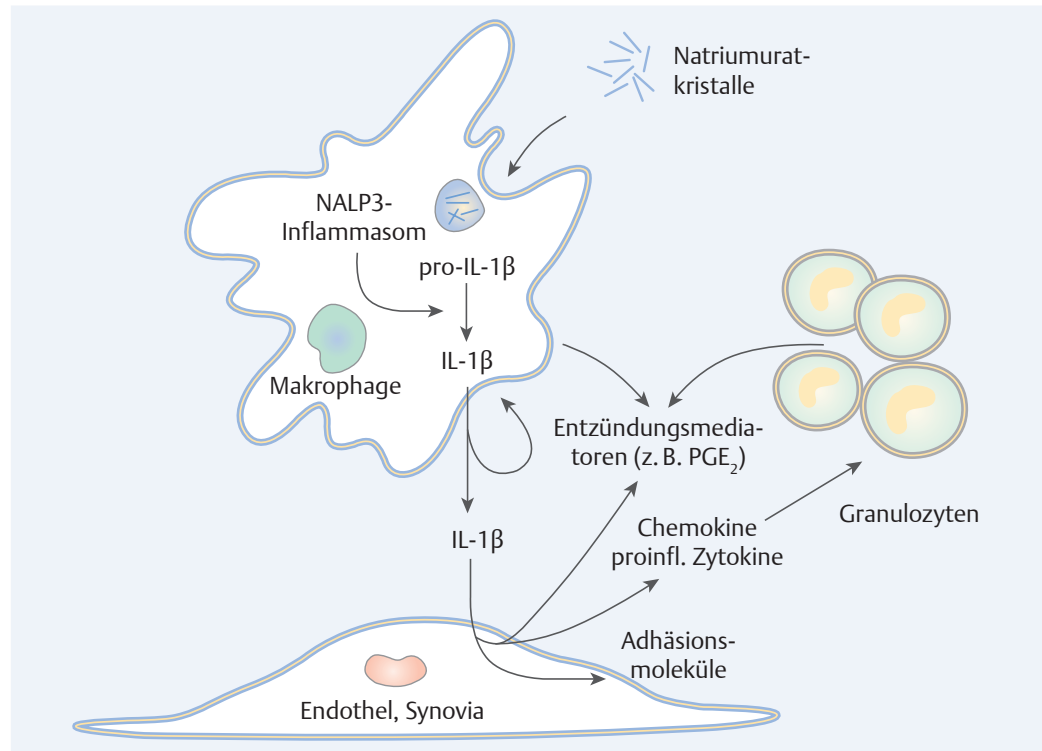

Abb. 1 Mechanismen der Entzündung beim akuten Gichtanfall [5].

- der plötzliche Beginn aus voller Gesundheit,

- die enorme Schmerzhaftigkeit,

- die Beschränkung auf ein Gelenk sowie

- eine intensive entzündliche Reaktion, die über die Gelenkgrenzen hinausgeht ( Abb. 2).

Vorbestehende Strukturschäden begünstigen den Befall eines Gelenks [6]. Ein polyartikulärer Befall ist initial nur selten [7]. Die Schmerzen können so stark sein, dass der Patient den Druck der Bettdecke oder Erschütterungen im Raum kaum ertragen kann. Am häufigsten ist das Großzehengrundgelenk betroffen ( $\triangleright$ Tab. 2 ). Die Gichtanfälle können Stunden bis Tage, unter Umständen auch länger andauern.

Auslösende Faktoren | Einen Gichtanfall lösen

z.B. aus:

- Traumata,

- vermehrte Purinzufuhr (Festessen),

- Alkoholexzess,

- Arzneimittel (z.B. Saluretika, Zytostatika),

- Bestrahlungen,

- Operationen,

- Fastenkuren.

Abb. 2 Akuter Gichtanfall im Bereich des Großzehengrundgelenks.

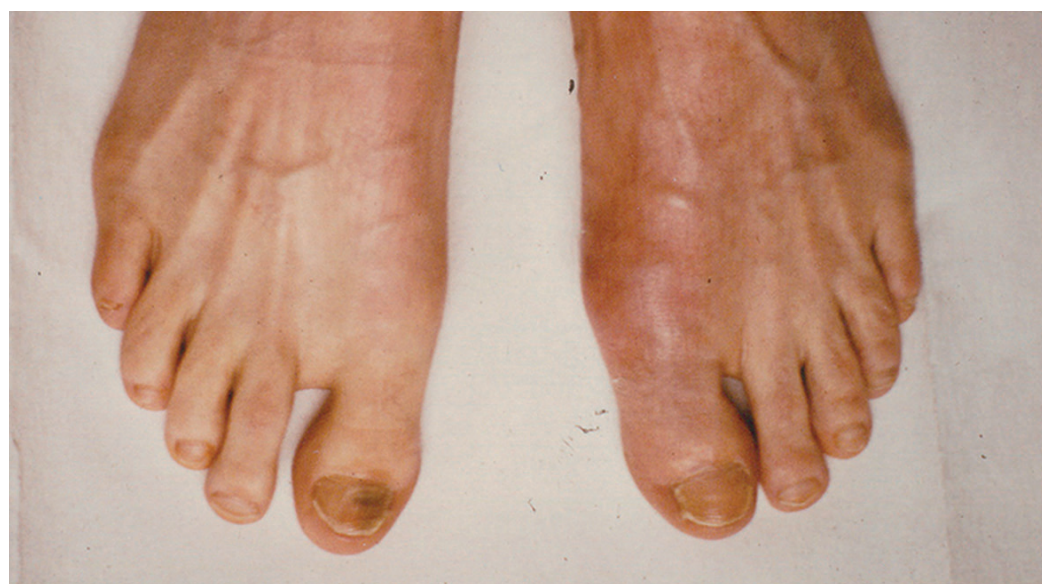




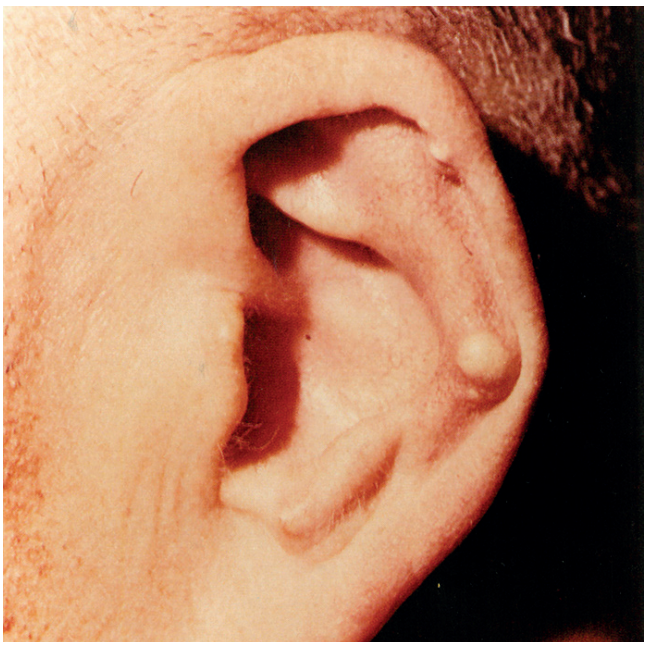

Abb. 3 Tophus im Bereich der Helix des Ohres („Gichtperle“).

Chronische Gicht | Ohne adäquate Behandlung wiederholen sich die Gichtanfälle und es werden nach und nach bis dahin noch nicht betroffene Gelenke befallen. In der Folge entwickelt sich eine chronische Gicht. Dieses Stadium ist durch Weichteil- und Knochentophi gekennzeichnet $($ Abb. 3 und $\triangleright$ Abb. 4). Die Gelenkveränderungen sind Folge der Zerstörung gelenknaher Knochenanteile durch Harnsäureablagerungen. Es kommt zu Deformierungen der Gelenke; Ankylosen sind selten.

Tophi bei chronischer Gicht| Weichteiltophi gehen meist vom periartikulären Gewebe, von Sehnenscheiden oder Schleimbeuteln aus. Gelegentlich brechen Tophi nach außen durch, es entsteht ein Gichtgeschwür. Selten können bei der Gicht auch neurologische Komplikationen, wie z.B. ein Karpaltunnel-Syndrom auftreten. Wir selbst beschrieben einen Patienten mit einer Paraparese beider Beine infolge eines Tophus im Bereich der LWS [8].

Niereninsuffizienz | Ein erhöhter Harnsäurewert ist ein Progressionsfaktor für eine Niereninsuffizienz. So sind Harnsäurewerte zwischen 7-9 mg/dl (420-540 $\mu \mathrm{mol} / \mathrm{l})$ gegenüber Harn-

Tab. 2 Häufigkeit des Befalls verschiedener Gelenke durch den ersten Gichtanfall [4].

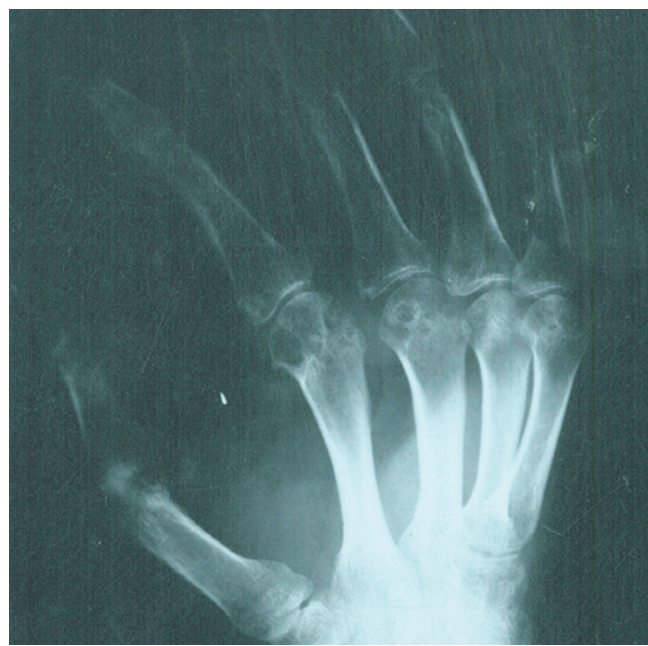

Abb. 4 Knochentophi.

säurewerten unter $7 \mathrm{mg} / \mathrm{dl}(420 \mu \mathrm{mol} / \mathrm{l})$ mit einem etwa zweifach erhöhten Risiko für eine Niereninsuffizienz verbunden [9].

Metabolisches Syndrom I Hyperurikämie und Gicht werden häufig im Rahmen eines metabolischen Syndroms beobachtet. Zahlreiche Studien weisen darauf hin, dass Hyperurikämie und insbesondere die Gicht mit einem erhöhten Risiko für koronare Herzkrankheit, Hypertonie und Herzinsuffizienz assoziiert sind [10-12].

\section{Diagnostik}

Vorgehen I Die Diagnose der Gicht beruht auf

- der typischen Anamnese,

- der Gelenkentzündung,

- ggf. dem raschen Ansprechen auf Colchicin,

- dem Nachweis von Uratablagerungen und

- einer Hyperurikämie [4].

Die Bestimmung der Serumharnsäure sollte aus dem Nüchternblut erfolgen (2-3 Bestimmungen an verschiedenen Tagen zur Bestätigung der Diagnose).

Bei einem akuten Gichtanfall kann jedoch der Serumharnsäurewert auch nur leicht oder nicht erhöht sein, weshalb dieser nach 2-3 Wochen erneut bestimmt werden sollte.

In den Tagen vor der Blutentnahme sollte sich der Patient wie gewohnt ernähren. Bei der Bewertung des Befunds muss die bisherige Arzneimitteltherapie berücksichtigt werden ( $\bullet$ Tab. 3 ). Ein Flussschema zum diagnostischen Vorgehen bei Hyperurikämie ist in $>$ Tab. 4 dargestellt.

24h-Urin | Bei jugendlichen Patienten, schwerer Verlaufsform oder rezidivierender Harnsäurenephrolithiasis sollte man neben der Serumharnsäure auch die Harnsäureausscheidung im 24h- 
Urin messen. Dies dient der weiteren Abklärung einer Hyperurikämie infolge eines Enzymdefekts des Purinstoffwechsels (Normalbereich unter Normalkost $500-600 \mathrm{mg} / \mathrm{die}$ ).

Gelenkbefall | Der Verdacht auf Gicht besteht bei jeder akuten Monarthritis des Erwachsenen - besonders, wenn eine schmerzhafte Gelenkschwellung vor Wochen, Monaten oder Jahren bereits einmal aufgetreten und abgeheilt ist. Das Gelenk ist geschwollen, gerötet ( $\bullet$ Abb. 2 ) und hochgradig schmerzhaft. Jede Bewegung, Erschütterung oder Berührung kann unerträglich sein.

Gelenkpunktion I Nur selten fehlen die allgemeinen Zeichen einer Entzündung. Rasches Ansprechen der Arthritis auf Colchicin bestätigt die Verdachtsdiagnose eines Gichtanfalls. In diagnostisch unklaren Fällen ist eine Gelenkpunktion mit dem Nachweis von Harnsäurekristallen in den polymorphkernigen Leukozyten der Gelenkflüssigkeit nützlich. Im Polarisationsmikroskop ist der Harnsäurekristall bei Rotpolarisation negativ doppelbrechend und parallel zur Kompensatorachse gelb, senkrecht dazu blau.

Sonografie | Auch die Gelenksonografie kann zur Diagnostik herangezogen werden. Dabei kann man beim akuten Anfall sonografisch eine Doppelkontur des Gelenkknorpels darstellen („Doppelkonturzeichen“). Auch intra- und extraartikuläre Tophi können mittels Ultraschall nachgewiesen werden.

Dual-Energy-CT I Eine weitere Methode ist die Dual-Energy-CT: Diese kann Harnsäureablagerungen in Gelenken, periartikulärem Gewebe und anderen Weichteilen sichtbar machen [13]. Die genaue Indikation zum Einsatz dieser Methode und ihr Stellenwert können derzeit noch nicht abschließend beurteilt werden.

Klinischer Score | In der hausärztlichen Praxis, in der meist keine Gelenkpunktion durchgeführt wird, kann zur Diagnose auch ein einfacher klinischer Score herangezogen werden ( $\bullet$ Tab. 5) [14].

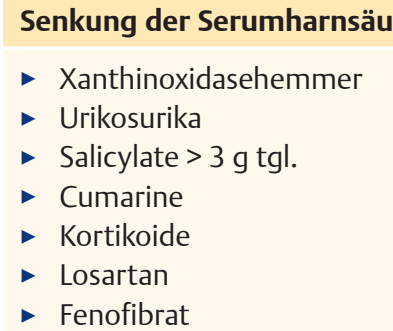

Nachweis von Tophi I Besondere diagnostische Bedeutung kommt auch dem Nachweis von Tophi zu, die in der Regel in einem fortgeschrittenen Stadium zu beobachten sind. Weichteilttophi finden sich vor allem im Bereich der Ohrmuscheln ( $\wedge$ Abb. 3), der Bursa olecrani und der Sehnenscheiden der Streckseite der Finger. Knochentophi finden sich bevorzugt im Bereich der Finger- und Großzehengrundgelenke ( Abb. 4).

Nieren I Wichtig bei Hyperurikämie und Gicht ist auch die Untersuchung der Niere.

\section{Differenzialdiagnosen}

Hyperurikämie | Differenzialdiagnostisch ist die familiäre Hyperurikämie von sekundären Formen abzugrenzen. Zum Nachweis eines angeborenen Stoffwechseldefekts dienen die Familienanamnese und Verwandtenuntersuchungen. Zum Nachweis sekundärer Hyperurikämieformen sollten ein vollständiges Blutbild angefertigt sowie Harnstoff, Kreatinin und Elektrolyte im Serum bestimmt werden. Weiterhin ist ein Harnstatus anzufertigen. Unerlässlich ist eine präzise Arzneimittelanamnese.

Gichtanfall | Im Vordergrund steht die Differenzialdiagnose der akuten Mono- oder Oligoarthritis. Nahezu alle rheumatischen Erkrankungen kön-
Tab. 3 Einfluss von Arzneimitteln auf die Serum-Harnsäurekonzentration.

Tab. 4 Flussschema zum diagnostischen Vorgehen bei Hyperurikämie.

\begin{tabular}{|c|c|c|c|}
\hline Eigenanamnese & Familienanamnese & klinischer Befund & Laboruntersuchungen \\
\hline $\begin{array}{l}\text { Plötzliche schmerzhaf- } \\
\text { te Gelenkschwellung? } \\
\text { Bursitis? } \\
\text { - Tendovaginitis? } \\
\text { Nierenkolik? } \\
\text { - Alkoholabusus? } \\
\text { Einnahme von } \\
\text { Serum-Harnsäure } \\
\text { erhöhenden Arznei- } \\
\text { mitteln, z.B. Diuretika? }\end{array}$ & $\begin{array}{l}\text { Gicht bei } \\
\text { männlichen } \\
\text { Blutsverwandten? } \\
\text { - Nephrolithiasis? } \\
\text { - Niereninsuffizi- } \\
\text { enz? }\end{array}$ & $\begin{array}{l}\text { - Akute Monarthri- } \\
\text { tis } \\
\text { - Tendovaginitis } \\
\text { - Bursitis } \\
\text { - Tophus } \\
\text { - Hypertonie }\end{array}$ & $\begin{aligned} & \text { Harnsäure im Serum } \\
& \text { und evtl. } 24 \text { h-Urin } \\
& \text { - } \text { Nierenstatus } \\
& \text { - } \text { Differenzialblutbild } \\
& \text { - } \text { Leberwerte } \\
& \text { - Blutzucker } \\
& \text { - Serum-Lipide }\end{aligned}$ \\
\hline
\end{tabular}

technische Untersuchungen

- Röntgenaufnahme des befallenen und kontralateralen Gelenks

- Röntgenaufnahme der Vorfüße (Tophi?)

- Gelenksonografie

- Dual-Energy-CT

- Computertomografie

- Kernspintomografie

- Nachweis von Harnsäurekristallen im Gelenk

- Ultraschall der Nieren 
Tab. 5 Klinischer Score zur Diagnose einer Gicht. Bei einer Punktzahl von 4-8 beträgt die Wahrscheinlichkeit des Vorliegens einer Gicht 32,2\%, bei einer Punktzahl von 8 und darüber $82,5 \%[14]$.

\section{Klinischer Score}

- männliches Geschlecht

2 Punkte

- Anamnese von einer oder

2 Punkte mehreren Episoden einer Monarthritis mit schmerzfreien Intervallen

- rasche Symptomentwicklung 0,5 Punkte innerhalb von $24 \mathrm{~h}$

- Befall des Großzehengrundgelenks

- Hautrötung über dem 1 Punkt betroffenen Gelenk

- Hypertonie oder mindestens 1,5 Punkte eine kardiovaskuläre Erkrankung

- Serumharnsäure $>5,88 \mathrm{mg} / \mathrm{dl}(353 \mu \mathrm{mol} / \mathrm{L})$
Nierenfunktionseinschränkung und Harnsäurenephrolithiasis | Die chronische Nierenfunktionseinschränkung bei Gicht reflektiert meist die Koexistenz anderer Erkrankungen wie Hypertonie, Diabetes und Arteriosklerose. Die Differenzialdiagnose betrifft die der chronischen Niereninsuffizienz. Die Differenzialdiagnose von Harnsäuresteinen umfasst in erster Linie die Differenzialdiagnose röntgennegativer Konkremente wie 2,8-Dihydroxyadenin- und Xanthin-Steinen.

\section{Therapie allgemein}

Harnsäurezielwert | Die Therapie der Gicht verfolgt zwei Ziele: einerseits die Behandlung des akuten Gichtanfalls und andererseits die dauerhafte Senkung der Serumharnsäure auf einen Harnsäurezielwert <6,0 mg/ dl $(360 \mu \mathrm{mol} / \mathrm{l})$.

Bei chronisch tophöser Gicht wird ein Harnsäurezielwert von $5,0 \mathrm{mg} / \mathrm{dl}$ (300 $\mu \mathrm{mol} / \mathrm{I})$ angestrebt.

nen in einer dieser beiden Formen beginnen. Ebenfalls in die Differenzialdiagnose einzubeziehen sind:

- Arthritis bei Gonorrhoe

- bakterielle Arthritis

- Pseudogicht

- Arthritis bei Viruserkrankungen

- aktivierte Arthrose

- Borreliose

Gezielte anamnestische Fragen zu Hautveränderungen (z.B. Psoriasis, Erythema nodosum, Erythema chronicum migrans), Durchfällen und Sehstörungen (Iridozyklitis) können differenzialdiagnostisch hilfreich sein.

Gelegentlich gehen Schmerzen, Schwellungen, Rötungen und Überwärmungen nicht von einem Gelenk aus, sind jedoch in der Nähe eines für einen Gichtanfall sehr typischen Gelenks lokalisiert.

Dies kann differenzialdiagnostische Probleme bereiten. So kann eine Phlegmone am medialen Vorfuß oder Fußrücken einem akuten Gichtanfall ähnlich sein. Ein weiteres Beispiel ist eine Bursitis an der Medialseite eines Großzehengrundgelenks, die durch mechanische Irritation hervorgerufen wurde oder ein Z.n. Trauma ist.

Tophus | Der Tophus ist differenzialdiagnostisch abzugrenzen von

- Rheumaknoten (rheumatoide Arthritis),

- Kalkknoten (Sklerodermie, Dermatomyositis),

- Xanthomen (familiäre Hypercholesterinämie),

- Fingerknöchelpolstern sowie

- Heberden-Knötchen (Fingerpolyarthrose).
Medikamente I Neben diätetischen Maßnahmen als Basistherapie stehen zur Harnsäuresenkung Arzneimittel zur Verfügung, die entweder die Harnsäurebildung hemmen (Xanthinoxidasehemmer) oder die renale Harnsäureausscheidung erhöhen (Urikosurika). Auch eine fixe Arzneimittelkombination aus $100 \mathrm{mg}$ Allopurinol und $20 \mathrm{mg}$ Benzbromaron ist auf dem deutschen Markt. Weitere harnsäuresenkende Arzneimittel sind die Rasburikase zur Prophylaxe und Therapie des Tumorlysesyndroms zu Beginn einer zytostatischen Therapie sowie die Pegloticase, einer pegylierten Urikase, bei schwerer therapierefraktärer, chronischer Gicht. Bei sekundärer Hyperurikämie und Gicht steht die Behandlung der Grundkrankheit im Vordergrund.

\section{Grundzüge der Ernährungstherapie} kämie und Gicht verfolgt folgende Ziele [15]:

- Verringerung der Purinzufuhr mit der Nahrung

- Normalisierung des Körpergewichts bei Übergewicht

- Einschränkung des Alkoholverbrauchs

- Ausreichende Flüssigkeitszufuhr

Purinzufuhr I Eine purinarme Kost sollte nicht mehr als 500 mg Harnsäure pro Tag bzw. 3000 mg pro Woche enthalten. Wir empfehlen unseren hyperurikämischen Patienten, nur einmal täglich Fleisch, Fisch oder Wurst (100-150g) zu essen und Innereien zu meiden. Auch Hülsenfrüchte sind purinreich. Bei den Ernährungsempfehlungen darf man nicht nur auf den Puringehalt eines Nahrungsmittels pro Gewichtseinheit achten, vielmehr ist auch der Puringehalt pro Energieein-
Ziele I Die Ernährungstherapie der Hyperuri- 
heit oder pro Portion zu berücksichtigen. Schmidt et al. fanden bei Männern die höchsten Serumharnsäurewerte bei Veganern, gefolgt von Fleischessern, Fischessern und Vegetariern [16].

Eiweißzufuhr I Den Eiweißbedarf (12-15\% kcal) sollte man bevorzugt mit fettarmen Milch- und Milchprodukten sowie mit Brot decken. Eine große Kohortenstudie konnte zeigen, dass der Genuss von fettarmen Milch- und Milchprodukten das Gichtrisiko senkt [17].

Fruktosezufuhr I Auch der Fruktosezufuhr in Form von mit Haushaltszucker gesüßten Getränken sowie Soft-Drinks wie Cola, Pepsi-Cola oder Sprite muss Aufmerksamkeit geschenkt werden. Choi et al. fanden in einer großen Kohortenstudie, dass mit steigendem Verbrauch dieser Getränke das Gichtrisiko zunimmt [18].

Übergewicht | Im Gegensatz zu Normalgewichtigen haben Übergewichtige höhere Serumharnsäurewerte. Die langsame Reduktion des Körpergewichts (max. 0,5-1 kg/Woche) ist deshalb ein weiteres wichtiges Ziel der Ernährungstherapie. Strenge Fastenkuren sind zu vermeiden, da sie über eine Ketonämie die renale Elimination der Harnsäure ungünstig beeinflussen.

Alkoholzufuhr | Unbedingt reduziert werden muss auch die Alkoholzufuhr. Alkohol verringert über eine vermehrte Laktatbildung die renale Harnsäureausscheidung. Außerdem führt es in größeren Mengen auch zu einer vermehrten Harnsäuresynthese. Schließlich ist auch der Puringehalt des Biers sowie der Kaloriengehalt des Alkohols zu berücksichtigen.

Flüssigkeitszufuhr | Nicht zuletzt sollte auch auf eine ausreichende Flüssigkeitszufuhr geachtet werden. Durch eine Wasserzufuhr von 21 oder mehr kommt die urikosurische Wirkung der Diurese zum Tragen.

\section{Medikamentöse harnsäuresenkende Therapie}

Indikation zur medikamentösen Therapie | Diätetische Maßnahmen sind die Basistherapie der Hyperurikämie und Gicht. Nach derzeitigen Leitlinien wird eine asymptomatische Hyperurikämie lediglich diätetisch bzw. durch Lebensstiländerungen behandelt.

Bei klinischen Manifestationen, wie z. B. Gichtanfällen und Tophi, ist eine zusätzliche medikamentöse harnsäuresenkende Therapie indiziert.

Diskutiert wird dabei, ob und bei welchen Patienten die medikamentöse Harnsäuresenkung be- reits nach dem ersten Gichtanfall erfolgen sollte. Die American College of Rheumatology sieht die Indikation gegeben, wenn die Gichtdiagnose gesichert ist und

- mindestens ein Tophus und/oder Gelenkzerstörungen nachgewiesen sind,

- eine chronische Niereninsuffizienz ab Stadium 2 (geschätzte glomeruläre Filtrationsrate unter $90 \mathrm{ml} /$ Min.) vorliegt oder

- der Patient eine Nephrolithiasis in der Anamnese aufweist [19].

Indikation nach EULAR I Die European League against Rheumatism (EULAR) empfiehlt in ihren neuen Leitlinien eine medikamentöse harnsäuresenkende Therapie sofort nach Diagnose einer symptomatischen Hyperurikämie [20].

Anfallsprophylaxe I Da zu Beginn einer medikamentösen harnsäuresenkenden Therapie gehäuft Gichtanfälle auftreten können, empfiehlt sich eine Anfallsprophylaxe mit niedrig dosiertem Colchicin (1-2 x 0,5 mg tgl.) bis zu 6 Monate lang. Alternativ kann man auch ein nichtsteroidales Antirheumatikum in niedriger Dosis einsetzen bei gastrointestinalen Risikofaktoren unter Zugabe eines Protonenpumpenhemmers [21].

Xanthinoxidasehemmer | Mittel der ersten Wahl sind Xanthinoxidasehemmer (Allopurinol, Febuxostat). In einigen Fällen besteht eine unbedingte Indikation zum Einsatz eines Xanthinoxidasehemmers, wie z. B. bei

- Harnsäurenephrolithiasis,

- Gicht infolge vermehrter Harnsäuresynthese,

- Adenin-Phosphoribosyltransferase-Mangel mit 2,8-Dihydroxyadenin-Lithiasis.

Nach der aktuellen Leitlinie des American College of Rheumatology ist Febuxostat dem Allopurinol gleichgestellt [19]. Die EULAR-Empfehlungen von 2014 empfehlen bei Gichtpatienten als Erstlinientherapie den Einsatz von Allopurinol [20]. Sollte unterAllopurinolderHarnsäurezielwert $<6 \mathrm{mg} / \mathrm{dl}$ (360 $\mu \mathrm{mol} / \mathrm{l})$ nicht erreicht werden, kommen als Alternativen in erster Linie

- Febuxostat oder

- ein Urikosurikum oder

- eine Kombination eines Xanthinoxidasehemmers mit einem Urikosurikum in Frage [19].

Allopurinol | Infolge der Hemmung der Xanthinoxidase fallen Serumharnsäure und renale Harnsäureausscheidung ab und die Ausscheidung von Hypoxanthin und Xanthin im Urin steigt gleichzeitig an. Allopurinol hemmt außerdem die Purinsynthese de novo sowie die Pyrimidinsynthese [4]. Die therapeutische Tagesdosis von Allopurinol liegt bei 200-300 mg. Man beginnt, z. B. nach Abklingen eines Gichtanfalls, mit einer Dosis von $100 \mathrm{mg}$ täglich. Wird der Harnsäurezielwert $<6,0 \mathrm{mg} / \mathrm{dl}(360 \mu \mathrm{mol} / \mathrm{l})$ unter niedriger Dosis nicht erreicht, steigert man in Abständen von zwei 
Wochen die Tagesdosis bis auf $300 \mathrm{mg}$. In einzelnen Fällen kann man die Dosis weiter steigern.

Wegen der Gefahr einer generalisierten Allopurinol-Überempfindlichkeitsreaktion sollte jedoch eine Oxipurinol-Konzentration von $100 \mu \mathrm{mol} / \mathrm{l}(15,2 \mu \mathrm{g} / \mathrm{ml})$ im Serum nicht überschritten werden.

Bei eingeschränkter Nierenfunktion ist eine Dosisreduktion erforderlich. Wird der Harnsäurezielwert $<6 \mathrm{mg} / \mathrm{dl}(360 \mu \mathrm{mol} / \mathrm{l})$ nicht erreicht, empfiehlt sich z.B. ein Umsetzen auf Febuxostat - sofern keine Kontraindikationen vorliegen.

Nebenwirkungen von Allopurinol | Die häufigsten unerwünschten Wirkungen von Allopurinol betreffen gastrointestinale Störungen und Überempfindlichkeitsreaktionen. Insbesondere handelt es sich dabei um Hautreaktionen (ca. 4\%), die von

- Hautjucken und

- makulo-papulösen Exanthemen bis hin zu

- schweren Hautreaktionen wie Stevens-Johnson-Syndrom, toxische epidermale Nekrolyse und Erythema exsudativum multiforme

reichen [5, 22]. Das generalisierte AllopurinolÜberempfindlichkeitssyndrom wird meist beobachtet, wenn bei eingeschränkter Nierenfunktion die Allopurinol-Dosis nicht reduziert wird. Auch die gleichzeitige Verabreichung von Allopurinol und Thiaziden erhöht dieses Risiko. Gefährdet sind vor allem Patienten mit dem Nachweis von HLA-B5801 [19]. Dieser HLA-Subtyp wird vor allem bei Patienten mit asiatischer Herkunft angetroffen.

Interaktionen mit Allopurinol | Einige Interaktionen von Allopurinol mit anderen Arzneimitteln entstehen durch die Hemmung der Xanthinoxidase. 6-Mercaptopurin und Azathioprin werden über die Xanthinoxidase metabolisiert. Deshalb muss ihre Dosis um (nicht auf) etwa 75\% reduziert werden, wenn Allopurinol gleichzeitig gegeben wird. So kann man eine Zunahme unerwünschter Wirkungen von Azathiprin und 6-Mercaptopurin - insbesondere Knochenmarkstoxizität - vermeiden.

Nach Möglichkeit sollte man auf die gleichzeitige Therapie mit Azathioprin bzw. 6-Mercaptopurin und Allopurinol verzichten.

Allopurinol beeinflusst außerdem die Pharmakokinetik von Cumarinderivaten und führt auf diesem Weg zu einer Verstärkung der antikoagulatorischen Wirkung. Auch die Metabolisierung von Theophyllin wird durch Allopurinol gehemmt.

Febuxostat I Ein wirksamer Hemmer der Xanthinoxidase ist auch Febuxostat, ein Nicht-Purin-
Analog. Es hemmt die Xanthinoxidase selektiver und stärker als Allopurinol und wird sowohl hepatisch als auch renal eliminiert [23]. Die Plasmahalbwertszeit beträgt 5-8 Stunden. Die Standarddosis von Febuxostat beträgt $80 \mathrm{mg}$ tgl., bei NichtErreichen des Harnsäurezielwertes $<6 \mathrm{mg} / \mathrm{dl}$ (360 $\mu \mathrm{mol} / \mathrm{l}$ ) kann die Dosis auf $120 \mathrm{mg}$ tgl. erhöht werden. Der Patient nimmt die Tagesdosis unabhängig von der Nahrungsaufnahme auf einmal ein. Hervorzuheben ist, dass bei Niereninsuffizienz bis zu einer Clearance von $30 \mathrm{ml} / \mathrm{min}$ keine Dosisanpassung erforderlich ist. Verschiedene randomisierte, doppelblind geführte Phase-IIIStudien haben Febuxostat und Allopurinol miteinander verglichen. In der Confirms-Studie waren 65\% der Patienten mit leichter bis mittelschwerer Nierenfunktionsstörung eingeschlossen [24-26].

Febuxostat erwies sich dabei in den zugelassenen Dosierungen und bei allen untersuchten Patientengruppen hinsichtlich der harnsäuresenkenden Wirkung dem Allopurinol überlegen.

Nebenwirkungen von Febuxostat | Die häufigsten Nebenwirkungen von Febuxostat sind

- Leberfunktionsstörungen,

- Durchfall,

- Übelkeit und

- Hautausschläge, die jedoch meist leicht- bis mittelschwer ausgeprägt waren [24-26].

Auch einzelne Fälle von Hypersensitivitätsreaktionen sind beobachtet worden.

Interaktionen mit Febuxostat | Arzneimittelinteraktionen von Febuxostat sind seltener als von Allopurinol. Aufgrund seines Wirkmechanismus sollte Febuxostat nicht gleichzeitig mit Azathioprin oder 6-Mercaptopurin verabreicht werden. Die gleichzeitige Verordnung von Febuxostat und Theophyllin ist möglich.

Kontraindikationen I Febuxostat wird derzeit vom Hersteller bei Patienten mit koronarer Herzkrankheit und dekompensierter Herzinsuffizienz nicht empfohlen - die Sicherheit ist in dieser Patientengruppe noch nicht durch eine klinische Studie abgesichert. Eine derartige klinische Studie ist in Gang (FAST: Febuxostat vs Allopurinol Streamlined Trial).

Vorteile von Febuxostat | Eine Untersuchung mit kleiner Fallzahl über insgesamt ein Jahr bei Patienten mit schwerer chronisch tophöser Gicht konnte jedoch zeigen, dass Febuxostat im Gegensatz zu Allopurinol einen günstigen Effekt auf die Gefäßsteifigkeit ausübt [27]. Während die Pulswellengeschwindigkeit zwischen Carotis und Femoralis in der Febuxostat-Gruppe unverändert blieb, nahm sie unter Allopurinol zu. Auch TNFalpha und die Aktivität der NADPH-Oxidase, einem Marker für den oxidativen Stress, nahmen in 
der Febuxostat-Gruppe ab. Unter Allopurinol blieben sie unbeeinflusst. Eine weitere Studie, die NUFlash-Studie, kam zu ähnlichen Ergebnissen [28].

Urikosurika | Den tubulären Harnsäuretransport hemmen Urikosurika - und damit in erster Linie die tubuläre Harnsäurerückresorption. Es kommt dadurch bis zur Einstellung eines neuen Gleichgewichts zu einer vermehrten renalen Harnsäureausscheidung. Dadurch entsteht die Gefahr von tubulären Harnsäureausfällungen. Urikosurika müssen deshalb einschleichend dosiert werden; gleichzeitig muss man auf eine ausreichende Diurese (Urinvolumen von 1,5-2,01/24h) und Harnneutralisierung (angestrebter Urin-pH 6,46,8) achten. In Deutschland stehen als Urikosurika Benzbromaron und Probenecid zur Verfügung:

Benzbromaron I Aufgrund seiner protrahierten Wirkung können Patienten die gesamte Tagesdosis von Benzbromaron als morgendliche Einzeldosis einnehmen. Die Therapie wird einschleichend begonnen, die Tagesdosis beträgt $25-100 \mathrm{mg}$. Die häufigsten unerwünschten Wirkungen sind gastrointestinale Störungen und allergische Reaktionen. Schwere Leberschäden sind beschrieben [5].

Probenecid I In einer Dosierung von 2-3g tgl. wird Probenecid verabreicht. Dabei ist eine Aufteilung der ermittelten Tagesdosis auf 2-3 Einzeldosen erforderlich, um größere Schwankungen der renalen Harnsäureausscheidung zu vermeiden. Die Behandlung erfolgt einschleichend. Unerwünschte Wirkungen treten bei Probenecid selten auf, wobei vor allem gastrointestinale Störungen und allergische Reaktionen beobachtet werden.

Zweitlinientherapie | Urikosurika gelten heute als Medikamente der Zweitlinientherapie bei der Harnsäuresenkung.

Sie sollten in der Regel nur noch bei GichtPatienten mit normaler Harnsäureausscheidung und ohne jegliche renale Symptomatik eingesetzt werden.

Urikosurika können mit Xanthinoxidasehemmern kombiniert werden und kommen z.B. zum Einsatz, wenn Xanthinoxidasehemmer nicht vertragen werden oder kontraindiziert sind.

Rasburikase | Als rekombinantes Enzym steht Rasburikase zur Verfügung - es katalysiert die Oxidation von Harnsäure zu dem wesentlich besser löslichen Allantoin. Rasburikase ist ein Urikolytikum, das für die Prophylaxe und Behandlung akuter Hyperurikämien zugelassen ist. Eine weitere Indikation besteht für Patienten mit hämatologischen Malignomen und hoher Tumorlast, bei denen nach Beginn einer Chemotherapie die Ge- fahr eines Tumorlyse-Syndroms besteht - hier kann Rasburikase ein akutes Nierenversagen verhindern. Die empfohlene tägliche Einmaldosis für das Enzym beträgt $0,2 \mathrm{mg} / \mathrm{kg} / \mathrm{KG}$ in Form einer intravenösen Infusion. Die Behandlung erstreckt sich über 5-7 Tage. Als unerwünschte Wirkungen können

- Fieber,

- Übelkeit,

- Erbrechen und

- allergische Reaktionen auftreten.

Pegloticase I Eine pegylierte Form der Rasburikase, die Pegloticase, wird bei schwerer therapierefraktärer Gicht eingesetzt. Dieses Medikament ist derzeit in Deutschland nicht zugelassen.

\section{Therapie des Gichtanfalls}

Frühzeitiger Beginn | Ziel der Behandlung des akuten Gichtanfalls ist die rasche Besserung der Gelenkentzündung und der damit einhergehenden Symptome. Die Behandlung sollte man so früh wie möglich innerhalb von $24 \mathrm{~h}$ nach Symptombeginn einleiten [20, 29]. Falls der Patient bereits eine harnsäuresenkende Therapie erhält, sollte diese unverändert fortgeführt werden.

Medikamente I Neben der Kühlung des akut entzündeten Gelenks und Ruhigstellung stehen zur medikamentösen Therapie

- nichtsteroidale Antirheumatika,

- Colchicin und

- Glukokortikoide

zur Verfügung [20, 21, 29]. Bei schweren Gichtattacken, polyartikulärem Befall und mangelndem Ansprechen auf eine Mono-Therapie ist auch eine kombinierte Arzneimitteltherapie möglich. Empfohlen wird dabei eine Kombination von nichtsteroidalen Antirheumatika oder Glucocorticoiden mit Colchicin [29].

Nichtsteroidale Antirheumatika | Nichtsteroidale Antirheumatika werden eingesetzt bei:

- akutem Gichtanfall

- normaler Nierenfunktion

- Fehlen von Kontraindikationen

Es werden in der Regel hohe Tagesdosen benötigt. Geeignet sind z.B. Diclofenac (150 mg tgl.), Ibuprofen (1800-2400 mg tgl.) oder Indometacin (100-150 mg tgl.). Bei gastrointestinalen Risikofaktoren sollte man zusätzlich Protonenpumpenhemmer verabreichen. Auch das Coxib Etoricoxib in der Dosis von $120 \mathrm{mg}$ tgl. steht zur Behandlung des akuten Gichtanfalls zur Verfügung. Die Behandlung erstreckt sich über einige Tage.

Colchicin | Traditionell wurden früher höhere Dosen von $4-8 \mathrm{mg}$ Colchicin innerhalb der ersten $24 \mathrm{~h}$ eingesetzt - heute ist die Dosis niedriger. Die American College of Rheumatology empfiehlt eine Initialdosis von $1,2 \mathrm{mg}$, gefolgt von einer 


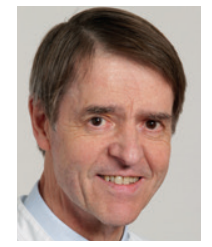

Prof. Dr. med. Wolfgang Gröbner ist an der Inneren Medizin der Kreisklinik Wertingen tätig. Wolfgang.Groebner@ khdw.de zweiten Dosis von 0,6 mg 1 Stunde später [29]. Da in manchen Ländern nur 0,5 mg-Tabletten zur Verfügung stehen, betragen die entsprechenden Dosen 1,0 bzw. 0,5 mg.

Die Therapie sollte innerhalb von 12-24h nach Symptombeginn eingesetzt werden. Bei späterem Einsatz sind die Erfolgsaussichten geringer.

Alternativ kann Colchicin auch in einer Dosis von $3 \times 0,5 \mathrm{mg}$ am ersten Tag eingesetzt werden [21]. Anschließend sollte die Behandlung mit 2-3×0,5 mg Colchicin tgl. weitergeführt werden. Dies gilt besonders dann, wenn eine medikamentöse harnsäuresenkende Therapie eingeleitet wird (Colchicinprophylaxe). Bei Nierenfunktionseinschränkung ist eine Dosisreduktion erforderlich, bei schwerer Niereninsuffizienz ist Colchicin kontraindiziert. Die häufigsten unerwünschten Wirkungen sind Übelkeit, abdominelle Schmerzen und Durchfälle.

Glukokortikoide I Systemisch oder intraartikulär können Glukokortikoide verabreicht werden. Die intraartikuläre Applikation kommt infrage, wenn der Gichtanfall 1-2 große Gelenke betrifft [21, 29]. Vorab muss eine bakterielle Arthritis sicher ausgeschlossen sein. Bei oraler GlukokortikoidGabe beträgt die empfohlene Dosis 0,5 mg Prednison je Kilogramm Körpergewicht und Tag [21, 29]. Sie sollte über 5-10 Tage verabreicht und dann abgesetzt werden. Alternativ ist die Verabreichung der vollen Dosis über 2-5 Tage mit einem Ausschleichen über 7-10 Tage.

Inhibitoren von Interleukin (IL)1-Beta | Nur wenn Gichtanfälle auf die übliche Therapie nicht ansprechen, kommen Inhibitoren von Interleukin (IL)1-Beta infrage. Zur Verfügung stehen Anakinra, Canakinumab und Rilonacept. Von diesen drei Substanzen ist bislang von der Europäischen Arzneimittelbehörde nur der monoklonale Interleukin-1-Beta-Antikörper Canakinumab mit Einschränkungen für die Therapie der Gicht zugelassen. Es handelt sich um eine sehr teure Therapie.

\section{Therapie der Harnsäurenephrolithiasis}

Interessenkonflikt Der Autor gibt an, dass folgende Interessenkonflikte bestehen: Honorare für Fortbildungsvorträge Berlin-Chemie.

DOI 10.1055/s-0041-104420 VNR 2760512015147121814 Dtsch Med Wochenschr 2015; 140: 1615-1626 (c) Georg Thieme Verlag KG . Stuttgart · New York . ISSN 0012-0472

Konservativ I Die konservative Therapie der Harnsäurenephrolithiasis umfasst:

- die Verabreichung eines Xanthinoxidasehemmers

- die Harnneutralisierung durch Alkali-Zufuhr (z.B. Uralyt-U, angestrebter Urin-pH 6,4-6,8)

- Maßnahmen zur Diuresesteigerung

\section{Prognose}

Gute Prognose I Im Allgemeinen ist die Prognose der Gicht gut. Unter konsequenter harnsäuresenkender Therapie sind die Patienten nach wenigen Monaten anfallsfrei. Weichteiltophi verschwinden, Knochentophi können sich unter Wiederherstellung des Gelenks ebenfalls zurückbilden. Unter Xanthinoxidasehemmern können sich Harnsäuresteine auflösen.

Eine rechtzeitige Therapie mit einer Senkung der Serum-Harnsäure auf einen Zielwert $<6 \mathrm{mg} / \mathrm{dl}(360 \mu \mathrm{mol} / \mathrm{L})$ verhindert das Auftreten von klinischen Manifestationen einer Hyperurikämie.

\section{Konsequenz für Klinik und Praxis}

- Ursache der Gicht ist die Hyperurikämie. Sie beruht bei der Mehrzahl aller Patienten auf einer Störung der tubulären Harnsäuresekretion.

- Die Diagnose der Gicht beinhaltet die Anamnese, den Nachweis von Harnsäureablagerungen und ggf. das Ansprechen auf Colchicin während des Anfalls sowie einer Hyperurikämie.

- Hyperurikämie und Gicht sind mit einem erhöhten Risiko für koronare Herzkrankheit, Hypertonie und Herzinsuffizienz assoziiert.

- Zur Behandlung des akuten Gichtanfalls eignen sich nichtsteroidale Antirheumatika, Colchicin sowie Glucocorticoide.

- Ziel der Langzeit-Therapie ist die Senkung der Serum-Harnsäure auf einen HarnsäureZielwert<6,0 mg/dl $(360 \mu \mathrm{mmol} / \mathrm{I})$. Bei fortgeschrittener chronisch-tophöser Gicht wird ein Wert von 5,0 mg/dl (300 ummol / I) angestrebt.

- Diätetische Maßnahmen bzw. Lebensstiländerungen sind die Basistherapie der Hyperurikämie und Gicht - und nach derzeitigen Leitlinien bei asymptomatischer Hyperurikämie allein indiziert.

- Unter den harnsäuresenkenden Arzneimitteln bei symptomatischer Hyperurikämie sind Xanthinoxidasehemmer das Mittel der ersten Wahl.

\section{Literatur}

1 Annemas L, Spaepen E, Gaskin M et al. Gout in the UK and Germany: Prevalence, comorbidities and management in general practice 2000-2005 Ann Rheum Dis 2008; 67: 960-966

2 Gröbner W, Bantel E. Hyperurikämie - häufige Begleiterkrankung beim metabolischen Syndrom. In: Mehnert H, Hrsg. Herz, Gefäße und Diabetes. Oberhausen: Medikon; 1997: 105-116 
3 Koettgen A, Albrecht E, Teumer A et al. Genomewide association analysis identify 18 new loci associated with serumurate concentrations. Nature Genetics 2013; 45: 145-154

4 Gröbner W, Gross M, Zöllner N. Krankheiten durch Störungen des Purin- und Pyrimidin-Stoffwechsels. In Gerok W, Huber C, Meinertz T, Zeidler H, Hrsg. Die Innere Medizin. 11. Aufl. Stuttgart: Schattauer; 2007: 1140-1151

5 Ludwig A, Gröbner W. Purinstoffwechsel - Gicht. In: Pharmakologie und Toxikologie. 11. Aufl. Berlin: Elsevier; 2013: 559-567

6 Roddy E, Zhang W, Doherty M. Are joints affected by gout also affected by osteoarthritis? Ann Rheum Dis 2007; 66: 1374-1377

7 Tausche AK, Manger B, Müller-Ladner U et al. Die Gicht als Systemerkrankung. Z Rheumatol 2012; 71: 224-230

8 Wallmüller-Strycker A, Walter B, Gröbner W et al. Zwei seltene neurologische Komplikationen bei Gicht. 19. Tagung Dtsch Ges Rheumatol 1980: 412

9 Obermayr RP, Temml C, Gutjahr G et al. Elevated uric acid increases the risk for kidney disease. J Am Soc Nephrol 2008; 19: 2407-2413

10 Fang J, Aldermann MH. Serum uric acid and cardiovascular mortality: The NHANES epidemiologic follow-up study 1971-1992. JAMA 2000; 283: 2404-2410

11 Stack AG, Hanley A, Casserly LF et al. Independent and conjoint associations of gout and hyperuricemia with total and cardiovascular mortality QJM 2013; 106: 647-658

12 Bhole V, Krishnan E. Gout and the heart. Rheum Dis Clin N Am 2014; 40: 125-143

13 Choi HK, Bruns LC, Shojanic K et al. Dual Energy CT in gout; a prospective validation study. Ann Rheum Dis 2012; 71: 1466-1471

14 Janssens HJ, Fransen J, van de Lisdonk EH et al. A diagnostic rule for acute gouty arthritis in primary care without joint fluid analysis. Arch Int Med 2010; 170: 1120-1126

15 Zöllner N, Gröbner W. Gicht und erhöhte Harnsäure. 9. Aufl. Stuttgart: Hirzel; 2013

16 Schmidt JA, Crowe FL, Appleby PN et al. Serum uric acid concentrations in meat eaters, fish eaters, vegetarians and vegans; cross-sectional analysis in the EPIC-Oxford cohort. PLOS one 2013; 8: e56339

17 Choi HK, Atkinson K, Karlson EW et al. Purine rich foods, dairy and protein intake and the risk of gout in men. N Engl J Med 2004; 350: 1093-1103

18 Choi HK, Curhan G. Soft drinks, fructose consumption and the risk of gout in men; prospective cohort study. Br Med J 2008; 336: 309-312
19 Khanna D, Fitzgerald JD, Khanna PP et al. American College of Rheumatology guidelines for management of gout. Part I. Systemic non pharmacologic and pharmacologic approaches to hyperuricemia. Arthritis Care and Res 2012a; 64: 1431-1446

20 Richette P, Doherty M, Pascual E et al. Updated EULAR evidence based recommendations for the management of gout. Ann Rheum Dis 2014; 73 : Suppl. 2

21 Zhang W, Doherty M, Bardin T et al. EULAR evidence based recommendations for gout. Part II: Management report of a task force of the EULAR standing committee for international clinical studies including Therapeutics. Ann Rheum Dis 2006; 65: 1312-1324

22 Miederer SE, Miederer KO. Allopurinol-Hypersensitivitäts-Syndrom: Lebertransplantation nach Behandlung einer asymptomatischen Hyperurikämie. Dtsch Med Wochenschr 2014; 139: 2537 2540

23 Edwards NL. Febuxostat: a new teatment for hyperuricemia in gout. Rheumatology 2009; 48 : ii15-ii19

24 Becker MA, Schumacher HR, Worthmann RL et al. Febuxostat compared with allopurinol in patients with hyperuricemia and gout. N Engl J Med 2005; 353: 2450-2461

25 Schumacher HR, Becker MA, Worthmann RL et al. Effects of febuxostat versus allopurinol and placebo in reducing serum urate in subjects with hyperuricemia and gout. A 28 Week Phase III, randomized, double blind, parallel-group trial. Arthritis \& Rheumatism 2008; 59: 1540-1548

26 Becker MA, Schumacher HR, Espinoza LR et al. The urate lowering efficacy and safety of febuxostat in the treatment of the hyperuricemia of gout; the confirms trial. Arthritis Res Ther 2010; 12: R63

27 Tausche AK, Christoph M, Forkmann M et al. As compared to allopurinol urate-lowering therapy with febuxostat has superior effects on oxidative stress and pulse wave velocity in patients with severe chronic tophaceous gout. Rheumatol Int 2014; 34: 101-109

28 Sezai A, Soma M, Nakata K et al. Comparison of febuxostat and allopurinol for hyperuricemia in cardiac surgery patients (NU-Flash-Trial). Circ J 2013; 77: 2043-2049

29 Khanna D, Fitzgerald JB, Khanna PP et al. American College of Rheumatology guidelines for the management of gout. Part II. Therapy and antiinflammatory prophylaxis of acute gouty arthritis. Arthritis Care Res 2012; 64: 1447-1461 


\section{CME-Fragen}

CME-Teilnahme

- Viel Erfolg bei Ihrer CME-Teilnahme unter http://cme.thieme.de

- Diese Fortbildungseinheit ist 12 Monate online für eine CME-Teilnahme verfügbar.

- Sollten Sie Fragen zur Online-Teilnahme haben, unter http:// cme.thieme.de/hilfe finden Sie eine ausführliche Anleitung.

1. Wie ist eine Hyperurikämie definiert (unter Berücksichtigung des Löslichkeitsprodukts von Natriumurat)?

a Serumharnsäure über $8,5 \mathrm{mg} / \mathrm{dl}(510 \mu \mathrm{mol} / \mathrm{l})$

b Serumharnsäure über $8,0 \mathrm{mg} / \mathrm{dl}(480 \mu \mathrm{mol} / \mathrm{l})$

c Serumharnsäure über $7,8 \mathrm{mg} / \mathrm{dl}(468 \mu \mathrm{mol} / \mathrm{l})$

d Serumharnsäure über $6,4 \mathrm{mg} / \mathrm{dl}(384 \mu \mathrm{mol} / \mathrm{l})$

e Serumharnsäure über $5,0 \mathrm{mg} / \mathrm{dl}(300 \mu \mathrm{mol} / \mathrm{l})$

2. Welches ist die häufigste klinische Manifestation einer Hyperurikämie?

a Tophus

b Nierenkolik

c Arthritis

d Bursitis

e Tendovaginitis

3. Welches Zytokin spielt beim akuten Gichtanfall eine wichtige Rolle?

a Interleukin 6

b Interleukin 2

c Interleukin 1-Beta

d Interleukin 10

e Interleukin 17

4. Welche Therapie ist bei asymptomatischer Hyperurikämie indiziert?

a Gabe niedrig dosierten Colchicins

b Verordnung von Allopurinol

c Verordnung von Febuxostat

d Ernährungsberatung

e Verabreichung eines Urikosurikums

5. Welches Gelenk ist beim ersten Gichtanfall am häufigsten betroffen?

a Sprunggelenk

b Daumensattelgelenk

c Großzehengrundgelenk

d Handgelenk

e Kniegelenk

6. Bei asymptomatischer Hyperurikämie sollte eine medikamentöse harnsäuresenkende Therapie bei folgenden Serumharnsäurespiegeln einsetzen:

a $\quad 6,0-6,9 \mathrm{mg} / \mathrm{dl}(360-414 \mu \mathrm{mol} / \mathrm{l})$

b $7,0-7,9 \mathrm{mg} / \mathrm{dl}(420-474 \mu \mathrm{mol} / \mathrm{l})$

c $\quad 8,0-8,9 \mathrm{mg} / \mathrm{dl}(480-534 \mu \mathrm{mol} / \mathrm{l})$

d über $9,0 \mathrm{mg} / \mathrm{dl}(540 \mu \mathrm{mol} / \mathrm{l})$

e Eine medikamentöse harnsäuresenkende Therapie ist nicht indiziert.

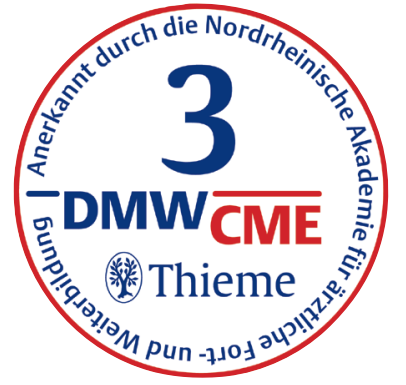

7. Bei einem Gichtpatienten mit eingeschränker Nierenfunkion (Kreatinin-Clearance $40 \mathrm{ml} / \mathrm{min}$ ) und Z.n. Harnsäurenephrolithiasis kann ohne Dosisreduktion folgendes harnsäuresenkendes Medikament zur Langzeittherapie verordnet werden:

a Benzbromeron

b Rasburicase

c Allopurinol

d Febuxostat

e Probenecid

8. Welche Arzneimittel werden heute zur langfristigen Harnsäuresenkung überwiegend eingesetzt?

a Xanthinoxidasehemmer

Urikosurika

Urikolytika

Interleukin 1-Beta-Inhibitoren

nichtsteroidale Antirheumatika

9. Welche Antwort ist richtig? Eine lebensgefährliche Knochenmarkaplasie kann sich rasch entwickeln...

a bei gleichzeitiger Gabe eines Xanthinoxidasehemmers und eines nichtsteroidalen Antirheumatikums.

b bei Kombination eines Xanthinoxidasehemmers mit Azathioprin.

c durch eine Interaktion von Probenecid und nichtsteroidalen Antirheumatika.

d unter längerfristiger Therapie mit Prednisolon.

e bei fehlender Dosisanpassung von Benzbromaron an die Nierenfunktion des Patienten.

\section{Welche Antwort ist richtig? Die Dosierung von Allopurinol} sollte nicht einschleichend erfolgen.

b darf $200 \mathrm{mg} / \mathrm{tgl}$. nicht überschreiten.

c muss bei eingeschränkter Nierenfunktion reduziert werden.

d ist nach dem Körpergewicht des Patienten zu berechnen.

e sollte stets auf drei Einzeldosen über den Tag verteilt werden. 


\section{Angaben zur Person}

Name, Vorname, Titel:

Straße, Hausnr.:

PLZ, Ort:

Anschrift: $\square$ privat $\square$ dienstlich

Ich bin Mitglied der Ärztekammer (bitte Namen der Kammer eintragen):

Jahr meiner Approbation:

Ich befinde mich in der Weiterbildung zum:

Ich habe eine abgeschlossene Weiterbildung in ... (bitte Fach eintragen):

Ich bin tätig als: $\square$ Assistenzarzt $\square$ Oberarzt $\square$ Chefarzt $\square$ niedergelassener Arzt $\square$ Sonstiges Ich bin DMW-Abonnent: $\square$ ja $\square$ nein Falls nein: ich habe den Fragebogen aus / von: $\square$ Thieme-connect $\square$ Kollegen $\square$ der Klinik $\square$ einer Bibliothek $\square$ Sonstiges

Lernerfolgskontrolle (Eine Antwort pro Frage ankreuzen)
1. $\square \mathrm{A} \quad \square \mathrm{B} \quad \square \mathrm{C} \quad \square \mathrm{D} \quad \square \mathrm{E}$
2. $\square$ A $\square$ B $\square$ C $\quad \square$ D $\quad \square \mathrm{E}$
3. $\square \mathrm{A} \quad \square \mathrm{B} \quad \square \mathrm{C} \quad \square \mathrm{D} \quad \square \mathrm{E}$
4. $\square$ A $\square$ B $\square$ C $\square$ D $\square$ E
5. $\square \mathrm{A} \quad \square \mathrm{B} \quad \square \mathrm{C} \quad \square \mathrm{D} \quad \square \mathrm{E}$
6. $\square$ A $\square$ B $\square$ C $\square$ D $\square \mathrm{E}$
7. $\square$ A $\square$ B $\square$ C $\square$ D $\quad \square$ E
8. $\square$ A $\square$ B $\square$ C $\square$ D $\square$ E
9. $\square \mathrm{A} \square \mathrm{B} \quad \square \mathrm{C} \quad \square \mathrm{D} \quad \square \mathrm{E}$
10. $\square \mathrm{A} \quad \square \mathrm{B} \quad \square \mathrm{C} \quad \square \mathrm{D} \quad \square \mathrm{E}$

Ich versichere, dass ich die Beantwortung der Fragen selbst und ohne fremde Hilfe durchgeführt habe

Ort, Datum:

Unterschrift:

Bitte in dieses Feld Ihre DMW Abonnement-Nummer eintragen:

\section{Fragen zur Zertifizierung}

1. Das Thema des Beitrages kommt in meiner ärztlichen Tätigkeit $\square$ häufig vor $\square$ selten vor $\square$ regelmäßig vor $\square$ gar nicht vor

2. Bei diesem Thema habe ich $\square$ eine feste Gesamtstrategie $\square$ keine Strategie $\square$ noch offene Einzelprobleme

3. In Bezug auf das Thema des Beitrages

$\square$ fühle ich mich nach dem Studium des Beitrags in meiner Strategie bestätigt

$\square$ habe ich meine Strategie verändert:

$\square$ habe ich erstmals eine einheitliche Strategie erarbeitet

$\square$ habe ich keine einheitliche Strategie ableiten können

4. Wurden aus der Sicht Ihrer täglichen Praxis heraus wichtige Aspekte des Themas

nicht erwähnt: $\square$ ja, welche $\square$ nein

zu knapp abgehandelt? $\square$ ja, welche $\square$ nein

überbewertet? $\square$ ja, welche $\square$ nein

5. Verständlichkeit des Beitrages

$\square$ Der Beitrag ist nur für Spezialisten verständlich

$\square$ Der Beitrag ist auch für Nicht-Spezialisten verständlich

6. Beantwortung der Fragen

$\square$ Die Fragen lassen sich aus dem Studium des Beitrags allein beantworten

$\square$ Die Fragen lassen sich nur unter Zuhilfenahme zusätzlicher Literatur beantworten

7. Die Aussagen des Beitrages benötigen eine ausführlichere Darstellung

$\square$ zusätzlicher Daten

$\square$ von Befunden bildgebender Verfahren

$\square$ die Darstellung ist ausreichend

8. Wieviel Zeit haben Sie für das Lesen des Beitrages und der Bearbeitung des Quiz benötigt?

Zertifizierungsfeld (wird durch die DMW ausgefüllt)

Ihr Ergebnis:

Sie haben von 10 Fragen richtig beantwortet.

Sie haben $\square$ bestanden und 3 Punkte erworben $\square$ nicht bestanden $\square$ ungültig, weil:

Stuttgart, den Stempel/Unterschrift 